\title{
Impacto de las microfinanzas en el bienestar e importancia de las pymes en la economía mundial
}

\author{
Luis Enrique Camacho Beas \\ Universidad de Lima \\ Ingeniería Industrial n. 28, 2010, ISSN 1025-9929, pp 61-83 \\ Recibido: 28 de enero del 2010 / Aprobado: 16 de febrero del 2010
}

RESUMEN: El objetivo de este artículo es mostrar, desde una perspectiva
conceptual y estadística, de qué manera la actividad microfinanciera
puede afectar el bienestar de las personas y las empresas, principalmente
de aquellas catalogadas como pymes. La idea principal radica en que el
proceso de facilitación de acceso al crédito permite ampliar las capacida-
des de consumo e inversión de estos agentes. Desde este punto de vista,
se entiende que la definición de bienestar estará centrada principalmente
en el análisis de la falta de acceso al crédito y a los productos financieros.
A partir del análisis estadístico se comprueba la importancia de las pymes
en la economía mundial y cómo estas son grandes generadoras de empleo.

Palabras clave: microfinanzas / pymes / bienestar

\section{The effect of microfinance in welfare and the importance of SME in the world economy}

ABSTRACT: The objective of this article is to demonstrate from a conceptual and statistic point of view how the microfinance activity can affect the wellbeing of the people and companies, mostly the ones under the SME's category. The main idea is that in the process of easing the access to credit allows the agent to expand its capacity of consume and investment. From this perspective, it is clear to understand that the definition of wellbeing will be mainly centered in the analysis of the lack of access to credit, but also in the analysis of the lack of access to financial products. This proves the importance of the SME's in the world economy and how these generate employment. The financial access becomes an important factor efficient development.

Keywords: microfinance / SME's / wellbeing 


\section{LAS MICROFINANZAS: CONCEPTOS PRELIMINARES}

La actividad microfinanciera no solo comprende la posibilidad de los individuos de tener acceso a un crédito, sino también implica generar acceso a productos financieros, como depósitos bancarios, diversas formas de ahorro en función al plazo o la moneda, acceso a medios electrónicos de pago y a seguridad. Es decir, las microfinanzas dan acceso a las personas y a la sociedad a una gama de servicios que van más allá del otorgamiento de créditos y que por lo tanto redundarán en la posibilidad de generar mejoras en su calidad de vida.

La investigación económica ha demostrado la sólida relación existente entre el desarrollo de la actividad financiera y el crecimiento económico; sin embargo, la mayor parte de estudios no analizan el impacto del acceso de las personas a las instituciones financieras, sobre todo en un escenario donde la relación entre demandante de servicios financieros e institución financiera se da bajo un esquema de nula o reducida experiencia financiera, por lo tanto los criterios que permiten medir el impacto del acceso al crédito podría describirse más como un cambio drástico en los patrones de comportamiento de los nuevos usuarios, a diferencia de aquellos que bajo el sistema tradicional de crédito no les es difícil o extraño acceder a él, por lo que tienen insumida dicha posibilidad dentro de sus opciones de generación de recursos.

Como se mencionó líneas arriba, hay un reconocimiento evidente del aporte de las instituciones financieras hacia el crecimiento económico. En este sentido, podemos mencionar la investigación pionera de Raymond Goldsmith, Financial structure and development (1969), donde se planteaba como objetivos documentar la forma como la estructura financiera cambia con el crecimiento económico, medir el impacto del desarrollo financiero en el crecimiento económico y establecer de qué manera la estructura financiera afectará el desarrollo de una economía. Otro investigador que ha aportado considerablemente al entendimiento de las instituciones financieras en el desempeño de las economías es Ross Levine, quien a lo largo de sus investigaciones llega a concluir que la dirección de la causalidad va del desarrollo financiero al crecimiento económico, logrando demostrar además que el desarrollo financiero es un buen predictor de las tasas de crecimiento de la economía. ${ }^{1}$

1 King y Levine (1993), para el periodo comprendido entre 1960-1989, analizan 77 países, encontrando que el desarrollo financiero es un buen predictor de las tasas de crecimiento 
Por otra parte, el estudio de las microfinanzas y su impacto en la actividad económica no tiene aún una literatura tan amplia como sí la tiene el estudio de las instituciones financieras, descrita anteriormente. Esto se debe principalmente a que las microfinanzas, en muchos casos, se basan en agentes que se encuentran fuera del canal económico formal, lo que hace que en términos reales el efecto en la mejora de la producción no se encuentre registrado en la información oficial de los países estudiados. Sin embargo, existen estudios que han dado luces sobre el impacto positivo de estas en la actividad económica local o regional. El trabajo de Sahidur Khandker (2003) concluye que:

[...] las microfinanzas tienen mucha significación para los muy pobres, así como para la economía local. Los programas microfinancieros tienen una particular significación para los pobres en el incremento del consumo per cápita, principalmente no alimenticio, así como de los activos familiares (excepto [la] tierra). Esto incrementa la probabilidad de que los participantes en los programas puedan ser capaces de salir de la pobreza. El impacto de las microfinanzas sobre el bienestar es también positivo para todos los hogares, incluyendo los de no participantes, lo que es indicativo de que los programas microfinancieros están ayudando a los pobres más allá de la redistribución del ingreso, al contribuir al crecimiento del ingreso local.

Por su parte, Romani Chocce (2002) argumenta lo siguiente:

[...] estos servicios llevan al cliente a modificar sus actividades microempresariales, lo cual lo lleva a aumentar/disminuir los ingresos de la microempresa. El cambio en los ingresos de la microempresa produce cambios en el ingreso del hogar, el cual lleva a una mayor/menor seguridad económica en el hogar. El nivel modificado de seguridad económica en el hogar lleva a cambios en los niveles educativos y de habilidades, y en las oportunidades económicas y sociales.

futuras de las economías. Levine y Zervos (1998), para el periodo comprendido entre 1976-1993, analizan 42 países y determinan que el desarrollo financiero es un buen predictor de las tasas de crecimiento posteriores de las economías. Levine, Loayza y Beck (1999) analizan 71 países para el periodo de 1960-1975 y determinan que la causalidad va desde el desarrollo financiero hacia el crecimiento y el efecto del primero sobre el segundo es económicamente significativo. Beck y Levine (2002) analizan para el periodo 1975-1998 a 40 países y determinan que la causalidad va desde el desarrollo financiero hacia el crecimiento y el efecto del primero sobre el segundo es económicamente significativo. 
En este caso se concluye el efecto multiplicador de los servicios financieros, logrando afectar las expectativas futuras de las familias y cambiando además de manera positiva sus patrones de comportamiento, sobre todo en lo referente al desarrollo de las capacidades de consumo y de formación de capital humano, lo que afectaría de manera importante la generación de ingresos potenciales, colaborando con el rompimiento del círculo de pobreza.

Según el estudio de John Weiss, Heather Montgomery y Elvira Kurmanalieva (2003), del Asian Development Bank Institute, el mecanismo por el cual las microfinanzas logran reducir la pobreza es bastante simple, ya que los pobres pueden financiar sus actividades productivas que les permitirán mejorar sus ingresos, estabilizándolos en el tiempo, evitando el efecto perverso de las fluctuaciones económicas y logrando mantener un consumo estable en el tiempo.

Una conclusión importante de estos estudios es el hecho de llegar a una alternativa de solución privada y de mercado al momento de buscar una solución al problema de la pobreza. Esto es así ya que las microfinanzas no se basan en esquemas asistencialistas, como suelen ser las políticas públicas de países de bajos ingresos, sino más bien son un tema de generación de oportunidades, pero lo más interesante es que la generación de dichas oportunidades son determinadas por agentes privados, es decir por prestatarios y prestamistas.

\subsection{Las microfinanzas desde la perspectiva del ofertante de servicios financieros}

En la literatura suelen confundirse los conceptos microcrédito y microfinanzas, considerándoseles lo mismo, cuando hay una marcada diferencia entre ambos. En primer lugar, el concepto microfinanzas puede definirse como la provisión de servicios financieros a agentes con limitados recursos. Es importante aclarar que no se está limitando el concepto de microfinanzas exclusivamente al crédito, así como tampoco se limita solo a personas pobres.

Desde esta perspectiva, las microfinanzas se pueden definir como la actividad que busca dar acceso a servicios financieros a los agentes económicos capaces de generar con su capacidad productiva, individual o colectiva, flujos suficientes para el pago de sus obligaciones bajo condiciones preferentes; las que están determinadas por su bajos niveles de capital y de empleo generado o ventas realizadas. 
La definición previa en ningún momento se desliga de la concepción original con la que se asocia a las instituciones microfinancieras con el acceso al crédito de los más pobres. Es más, la definición inicial incorpora un nuevo grupo de personas que se benefician también de este acceso al financiamiento sin ser necesariamente pobres.

En ese sentido, el microcrédito sería solo una parte de las microfinanzas. El desarrollo del sector microfinanciero empieza siempre con el desarrollo del microcrédito y continúa con la ampliación de otros servicios financieros. Las microfinanzas, entonces, involucran la provisión de servicios financieros por parte de las instituciones microfinancieras.

Los servicios financieros, por lo tanto, se dividen de la siguiente forma:

- Créditos.- Asociados directamente a la asignación de microcréditos destinados a determinados agentes, ya sean empresas o familias. Esta asignación de recursos se realiza previa evaluación de las posibilidades de pago del beneficiario.

- Ahorro, llamado también microahorro.- El microahorro es la captación de recursos por parte de las instituciones microfinancieras, que en gran parte es intermediario que genera microcréditos. Su característica principal radica en los bajos montos y bajas comisiones a que son afectos. En este caso, el microahorro genera adicionalmente la protección de los recursos escasos de sus ahorristas.

- Servicios de transferencias y pagos.- Servicios asociados directamente con las facilidades de la banca electrónica y el pago de servicios. La recepción de remesas es una situación común en las personas de bajos recursos, ya que no es difícil encontrar un familiar que haya migrado al exterior de manera que parte de los ingresos de las familias están constituidos por los ingresos por remesas. Este servicio tiende a tener menores costos que aquellos ofrecidos por las empresas especializadas en la transferencia de dinero.

- Seguros.- Es una protección contra determinados riesgos que se brinda a personas de bajos ingresos a cambio de pago regular de una prima. Las personas de bajos recursos son más vulnerables a los riesgos de enfermedad, desempleo, fallecimiento, por lo que el impacto de dichos riesgos es más frecuente. Este es el elemento menos desarrollado en el ámbito de las microfinanzas. 


\section{Gráfico 1}

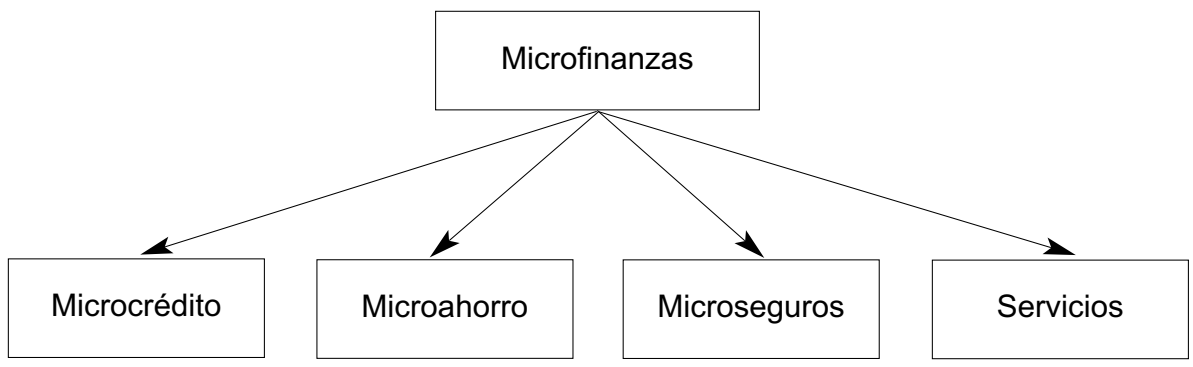

Elaboración propia.

Las microfinanzas son, por lo tanto, un conjunto de servicios financieros a los que los agentes, sean estos personas o empresas, pueden acceder de manera que les permita satisfacer sus necesidades de financiamiento, generación de ahorro y cobertura de riesgos.

\subsection{Análisis teórico de las mejoras sobre el bienestar como consecuencia del acceso al crédito}

En el análisis de las microfinanzas existen dos proposiciones complementarias. La primera postula que las microfinanzas pueden mejorar el bienestar de las personas de muy bajos ingresos, ya que estas permiten el acceso a productos financieros (ahorro y crédito) con los que antes no contaban; a este enfoque se le denomina el enfoque de la "restricción de liquidez". La segunda posición manifiesta que las personas de bajos ingresos no mejoran su bienestar por acceder a productos crediticios, ya que realmente sí tienen acceso a estos, pero son de carácter informal, y por lo tanto pueden tener acceso al crédito a tasas mucho más altas que las que podrían obtener si acceden al mercado microfinanciero formal.

El análisis económico de los beneficios del acceso a los servicios financieros se basa principalmente en los criterios microeconómicos utilizados para comprender los problemas de las "restricciones de liquidez". En el análisis económico se define como restricción de liquidez a la imposibilidad de contar con recursos necesarios para poder acceder a bienes y servicios en el mercado, de tal manera que se genera una restricción en las posibilidades de acceder al mercado; es en este esce- 
nario donde la teoría del consumo se asocia con la imposibilidad de incrementar las posibilidades de consumo de los agentes basados principalmente en los ingresos disponibles en los periodos que se están analizando. Desde esta perspectiva, y en un lapso de dos periodos, el conjunto factible del agente se limitaría a dos puntos fijos e inmóviles, por lo tanto la restricción presupuestaria de los agentes podría representarse de la siguiente manera:

$$
\mathrm{C}_{t}=\mathrm{F}\left(\mathrm{Yd}_{t}\right) \text {, para todo } t
$$

donde $\mathrm{C} t$ es Consumo en el periodo $t$ e $\mathrm{Yd} t$ es ingreso disponible en dicho periodo.

Como puede apreciarse, hay una relación directa entre el nivel de ingreso y el consumo en cada periodo, por lo que el consumo de los agentes estará limitado al nivel de ingreso del periodo y por lo tanto el consumo se verá afectado en gran medida con las fluctuaciones del ingreso.

Ampliaremos el análisis considerando ahora que las familias tienen la posibilidad de endeudarse, dado que cuentan con acceso al crédito. En este escenario las posibilidades de consumo se incrementan de manera importante, ya que ahora tendrán la siguiente restricción de presupuesto:

$$
\mathrm{Y}_{1}+\mathrm{Y}_{2} /(1+\mathrm{r})=\mathrm{C}_{1}+\mathrm{C}_{2} /(1+\mathrm{r})
$$

donde $\mathrm{Y}_{1}$ e $\mathrm{Y}_{2}$ son los ingresos de dos periodos consecutivos y $\mathrm{C}_{1} \mathrm{y}_{2}$ es el consumo en dos periodos consecutivos.

Este enfoque de carácter intertemporal permite reducir la dependencia del consumo a los ingresos del periodo, ya que ahora el nuevo conjunto factible de elección del individuo genera una mayor cantidad de combinaciones entre consumo presente y futuro. En este caso estamos frente a un escenario donde las posibilidades de consumo están en función del valor presente de los ingresos actuales y futuros. 
Gráfico 2.A

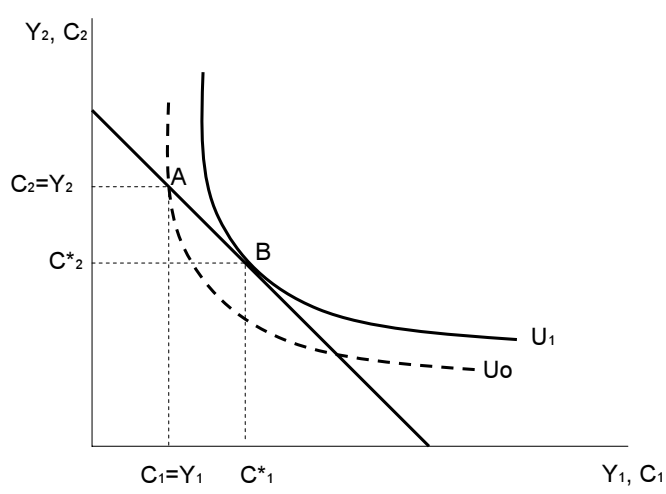

Elaboración propia.
Gráfico 2.B

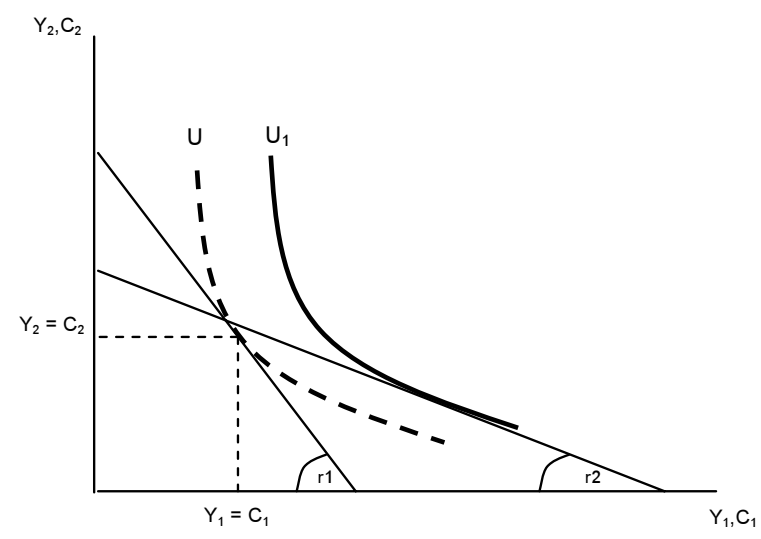

Elaboración propia.

En el gráfico 2.A podemos identificar el punto A como aquel asociado a una restricción de liquidez, es decir a la imposibilidad de acceder al mercado crediticio, donde el nivel de ingreso determina en buena medida el nivel de consumo de cada periodo. El punto B, por el contrario, se transforma en un punto de optimización, donde los individuos tienen acceso al mercado de crédito, permitiéndole acceder a una mayor variedad de combinaciones entre consumo presente y futuro, lo que se refleja en una mejora en el nivel de bienestar de los individuos, logrando alcanzar este agente una curva de indiferencia más alejada del origen. ${ }^{2}$

Otro punto de vista argumenta que las personas de bajos ingresos no mejoran su bienestar por acceder a productos crediticios, ya que realmente sí tienen acceso a estos pero son de carácter informal y, por lo tanto, pueden tener acceso al crédito a tasas muchos más altas que las que podrían obtener si acceden al mercado microfinanciero formal; así, la mejora en el bienestar de las personas y microempresas se asocia a la reducción de los costos de financiamiento, es decir, pasan de una tasa de interés $\mathrm{r} 1$ a r2, tal como se presenta en el gráfico 2.B.

2 Las curvas de indiferencia son una combinación de todos los puntos que dejan al individuo indiferente, en términos de bienestar. Las curvas de indiferencia, por sus propiedades de transitividad, reflexividad y completitud, garantizarán siempre el proceso de elección a través de la jerarquización de estas. 


\subsection{Análisis de las microfinanzas desde la perspectiva de la teoría de los contratos}

Según los estudios de Morduch (1999: 1569-1614), existen tres tipos de contratos que avalan el accionar de las instituciones microfinancieras, pudiendo dividirse en préstamo grupal, incentivos dinámicos y repago regular. El contrato grupal se define como aquel préstamo o crédito otorgado a una persona que forma parte de un grupo de personas cuya constitución es voluntaria y en donde todos sus integrantes son responsables de la cancelación, por lo que si una persona incurre en morosidad, entonces el resto del grupo tendrá la obligación de asumir su parte ante la institución prestadora. Lo importante de este tipo de contratos de crédito es que logra reducir en gran medida el nivel de riesgo del tomador del crédito al diversificarlo entre varios "avalistas".

El contrato de incentivos dinámicos consiste en comenzar la relación crediticia con pequeños préstamos iniciales, de manera que a medida que el beneficiario del crédito demuestra responsabilidad en el cumplimiento de sus obligaciones asumidas, posteriormente el crédito puede incrementarse.

Por último, el contrato con repagos regulares se refiere a que el prestatario debe comenzar a reembolsar el préstamo inmediatamente después de adquirirlo y realizar pagos frecuentes. Armendariz y Murdoch (2000: 401-420) establecen que a este tipo de contratos se le puede atribuir tres ventajas:

- Los individuos indisciplinados crediticiamente salen del sistema de manera rápida.

- Los demandantes de recursos dan señales rápidas a los prestamistas sobre los riesgos de los prestatarios.

- Permite a la institución financiera apropiarse del efectivo obtenido de los proyectos antes de que sea utilizado para el consumo u otros fines.

\section{MICROEMPRESA Y PEOUEÑA EMPRESA. ANÁLISIS ESTADÍSTICO DE SU IMPORTANCIA}

En el acápite anterior se llegaron a definir de manera adecuada las características de las microfinanzas actuales. En la mayor parte de las definiciones presentadas existe cierto consenso sobre el público objeti- 
vo y las carencias y necesidades de este; sin embargo, al momento de definir las microempresas, pequeñas empresas y medianas empresas no se encuentra un criterio único para su definición como tal. Lo que sí es cierto es que el principal criterio de clasificación se basa en el empleo generado más que en un criterio de ventas o ingresos.

En el siguiente cuadro se pueden apreciar los criterios de clasificación para micro, pequeñas, medianas y grandes empresas en Latinoamérica. Este cuadro se ha elaborado con la información recopilada en la investigación de Emilio Zevallos (2003). El cuadro se ha enriquecido con los criterios aplicados en el Perú.

\section{Cuadro 1}

\begin{tabular}{|c|c|c|c|c|c|}
\hline & Criterios & Microempresarios & $\begin{array}{l}\text { Pequeña } \\
\text { empresa }\end{array}$ & $\begin{array}{l}\text { Mediana } \\
\text { empresa }\end{array}$ & Gran empresa \\
\hline Argentina & Ventas & Hasta 0.5 & Hasta 3 & Hasta 24 & Más de 24 \\
\hline Bolivia & Empleo & hasta 10 & Hasta 19 & Hasta 49 & Más de 49 \\
\hline Chile & Ventas & Hasta 2400 & Hasta 25000 & Hasta 100000 & Más de 100000 \\
\hline Colombia & Empleo & Hasta 10 & Hasta 50 & Hasta 200 & Más de 200 \\
\hline Costa Rica & Empleo & Hasta 10 & hasta 30 & Hasta 100 & Más de 100 \\
\hline EI Salvador & Empleo & Hasta 4 & Hasta 49 & Hasta 99 & Más de 99 \\
\hline Guatemala & Empleo & Hasta 10 & Hasta 25 & Hasta 60 & Más de 60 \\
\hline México & Empleo & hasta 30 & Hasta 100 & Hasta 500 & Más de 500 \\
\hline Panamá & Ingresos & Hasta 150 mil & Hasta 1 millón & Hasat 2.5 millones & Más de 2.5 millones \\
\hline Venezuela & Empleo & Hasta 10 & Hasta 50 & Hasta 100 & Más de 100 \\
\hline Perú & Empleo & Hasta 10 & Hasta 50 & Hasta 250 & Más de 250 \\
\hline
\end{tabular}

Argentina

Bolivia

Chile

Colombia

Costa Rica

El Salvador

Guatemala

México

Panamá

Venezuela
Millones de pesos. Definición para la manufactura. Existen otras para sectores del comercio y servicios basados, además, en ventas anuales.

Aunque existen también criterios de ventas y activos, la definición oficial se basa en el empleo.

Expresado en UF. Una UF equivale aproximadamente a 24 dólares.

Dos definiciones, por empleo y activos.

Existen otras definiciones por inversión y por ventas anuales.

Las definiciones oficiales son por empleo y activos.

Definición del Instituto Nacional de Estadística.

Definición para la manufactura.

En balboas o dólares.

Definición aplicable solo a la manufactura.

Fuente: Instituciones oficiales de los respectivos países.

Elaboración propia. 
En los países miembros de la Organización para la Cooperación y el Desarrollo Económico (OCDE) ${ }^{3}$, (OECD por sus siglas en inglés) también hay una gran diferencia de criterios al definir los distintos tipos de empresa y, por lo tanto, la clasificación de una empresa como pequeña o microempresa. A continuación presentamos las definiciones en algunos países miembros de la OCDE.

\section{Cuadro 2}

\begin{tabular}{|c|c|}
\hline País & Criterio de clasificación \\
\hline Canadá & $\begin{array}{l}\text { - Menos de } 500 \text { empleados o utilidades menores a } 50 \text { millones de dólares } \\
\text { canadienses }\end{array}$ \\
\hline Japón & $\begin{array}{l}\text { - Empresa cuyo capital de inversión no supere los } 300 \text { millones de yenes } \\
\text { - Empresa con menos de } 300 \text { personas y cuya actividad principal sea } \\
\text { manufactura, construcción o transporte } \\
\text { - Para empresas del sector mayorista con capital de } 100 \text { millones de yenes } \\
\text { o como máximo } 100 \text { trabajadores } \\
\text { - Para empresas del sector servicios con menos de } 100 \text { trabajadores o con } \\
\text { inversión o capital menor a } 50 \text { millones de yenes }\end{array}$ \\
\hline Nueva Zelanda & $\begin{array}{l}\text { - Microempresas, de } 0 \text { a } 5 \text { empleados } \\
\text { - Pequeñas empresas, de } 6 \text { a } 20 \text { empleados } \\
\text { - Medianas, de } 21 \text { a } 50 \\
\text { - Grandes, más de } 50\end{array}$ \\
\hline Noruega & $\begin{array}{l}\text { - Empresas que cumplan dos de los siguientes criterios: } \\
\text { 1. Menos de } 40 \text { millones de coronas en utilidades } \\
\text { 2. Activo menor a } 20 \text { millones de coronas } \\
\text { 3. Menos de } 50 \text { empleados }\end{array}$ \\
\hline Turquía & $\begin{array}{l}\text { - Microempresa, de } 1 \text { a } 9 \text { empleados } \\
\text { - Pequeña empresa, de } 10 \text { a } 49 \text { empleados } \\
\text { - Mediana empresa, de } 50 \text { a } 249 \text { empleados }\end{array}$ \\
\hline Estados Unidos & $\begin{array}{l}\text { - Minería y manufactura, hasta } 500 \text { empleados } \\
\text { - Mayorista, hasta } 100 \text { empleados } \\
\text { - Empresas no productoras de bienes y con facturación de hasta } \\
\text { US\$ } 6 \text { millones }\end{array}$ \\
\hline
\end{tabular}

Fuente: OCDE.

Elaboración propia

3 La OCDE es un organismo internacional que ayuda a los gobiernos a afrontar los desafíos económicos, sociales y de gobierno en una economía global. El grupo de 30 países miembros, donde México es el único de Latinoamérica, comparten el compromiso por un Estado democrático y por la economía de mercado. La OCDE es mejor conocido por sus publicaciones y sus estadísticas, su trabajo cubre temas económicos y sociales desde la macroeconomía al intercambio, educación, desarrollo y ciencia e innovación. 
El desarrollo de estas empresas ha sido un fenómeno que se da en todos los países y su importancia en la actividad económica mundial se puede resumir en su aporte en la generación de empleo y en lo importantes que son en términos del total de empresas en cada país.

Las pequeñas y microempresas son protagonistas del impulso económico de la actividad productiva, no solo en los países pobres y de medianos ingresos. A continuación se presentan las características e importancia económica de dichas empresas en algunos países. Dado que la fuente de cada uno es distinta, las variables que se mencionan no necesariamente son coincidentes en todos los países seleccionados:

\section{Alemania:}

- 3,38 millones de empresas catalogadas como pymes.

- 19,9 millones de trabajadores trabajando en las pymes.

- Representan el 99,7\% del total de empresas.

- Generan el 70\% del empleo del sector privado.

- Capacitan al 82\% de la mano de obra.

- El 70\% tiene un solo propietario.

- Representan el 98\% de las empresas exportadoras.

- Representan el $21 \%$ del valor exportado.

\section{Brasil:}

- 4,8 millones de empresas catalogadas como pymes.

- Representan el 99\% del total de empresas.

- Generan el 56,2\% del empleo formal total.

- En el ámbito informal existen 9,5 millones de unidades productivas que podrían ser catalogadas como pymes.

\section{China:}

- Contribuyen con el $60 \%$ del PBI total.

- Generan el 75\% del empleo urbano.

- Principal destino de los despedidos por las privatizaciones de empresas.

\section{España:}

- 3,1 millones de empresas catalogadas como pymes.

- Representan el 99,8\% del total de empresas. 
- El 60\% es unipersonal.

- Generan el 89\% del empleo del sector privado.

- El $52 \%$ de las pymes se ubican en el sector servicios, $26 \%$ en el comercio, $14 \%$ en construcción y $8 \%$ en industria.

\section{Estados Unidos:}

- 22,9 millones de empresas catalogadas como pymes.

- Representan el 99,7\% del total de empresas.

- El 70\% es unipersonal.

- Crean el 70\% de los nuevos empleos anualmente.

- Aportan más del 50\% del PBI agrícola.

- Representan el 97\% de las empresas exportadoras.

- Representan el 29\% del valor exportado.

\section{Italia:}

- Son el 99\% de las empresas de la industria y servicios.

- Generan el 82\% del empleo total de la economía.

- Generan el 72\% del empleo industrial.

- Generan el $90 \%$ del empleo en la construcción y servicios.

Japón:

- 4,7 millones de empresas catalogadas como pymes.

- Representan el 99,7\% del total de empresas.

- Generan el 70,2\% del empleo total de la economía.

- Generan el 50\% del valor agregado de la industria y el 73\% del valor agregado del comercio.

De lo mostrado hasta ahora, podemos concluir que este sector empresarial no es un fenómeno exclusivo de las economías pobres ni de ingresos medios; por el contrario, estas tienen una participación muy importante en la actividad económica, incluso en las economías de los países industrializados.

A continuación analizaremos los criterios de clasificación de las microempresas, las pequeñas empresas y las medianas empresas, basándonos en la información proporcionada por la base de datos del Banco Mundial: "Micro, small, and medium enterprises: A collection of published data", elaborada por Mark Kozac, cuya última actualización se realizó el 2007. 
El Banco Mundial clasifica los países en función de sus ingresos, de la siguiente manera:

- Los países de ingreso alto son aquellos cuyo PBI per cápita es de US\$9.386 o más en 1995. El grupo incluye tanto a países desarrollados como a economías de ingresos altos en desarrollo.

- Los países de ingreso bajo son aquellos cuyo PBI per cápita es de US $\$ 765$ o menos en 1995.

- Los países de ingreso mediano son aquellos cuyo PBI per cápita era de US $\$ 766$ a US\$9.385 en 1995 y que a su vez se dividen en países de ingreso mediano bajo (US\$766-US\$3.035) y países de ingreso mediano alto (US\$3.036-US\$9.385).

Las características de los criterios de clasificación no son homogéneas, por lo que se procederá a realizar un análisis de las distintas clasificaciones en función de los ingresos de los países.

\subsection{Clasificación en países de ingresos altos}

En el gráfico 3 se observa cómo los países considerados dentro del grupo de países de ingresos altos han clasificado a las microempresas desde 1999 hasta el año 2005. Bajo este criterio, aproximadamente el 75\% de países de este grupo ha calificado como microempresas a aquellas con un número de trabajadores comprendidos entre 0 y 9 . El $25 \%$ restante de países clasifica como microempresas a aquellas que tienen entre 1 y 4 trabajadores.

En el gráfico 4 se aprecia la evolución en la definición de pequeña empresa en los países de altos ingresos. A diferencia de la definición de microempresa, en este caso existe una mayor variedad de definiciones, sin embargo la más considerada es aquella que define a una pequeña empresa como aquella que concentra entre 10 y 49 trabajadores, siendo seguidas en segundo lugar por las que agrupan entre 5 y 19 trabajadores.

En estos casos llama la atención la mayor disparidad de criterios encontrados al analizar las pequeñas empresas. De esta forma se infiere que al existir un criterio más homogéneo en el lado de las microempresas, este se ajusta a una política de desarrollo de dicho sector que es bastante común entre los países de la muestra, lo que no sucede con las pequeñas empresas, que al tener criterios de definición 


\section{Gráfico 3}

Países de ingresos altos: Definición de microempresa

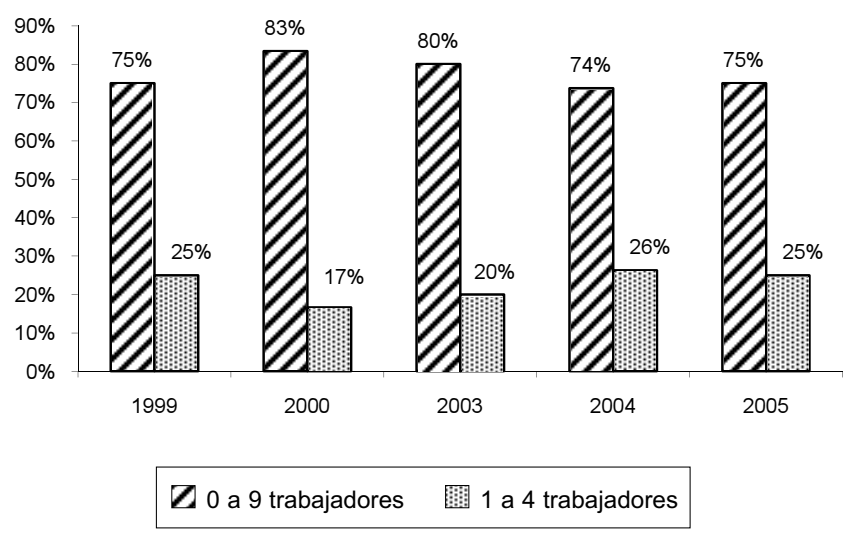

Fuente: "Micro, small and medium enterprises: A collection of published data" 2007. Elaboración propia.

\section{Gráfico 4}

Países de ingresos altos: Definición de pequeñas empresas

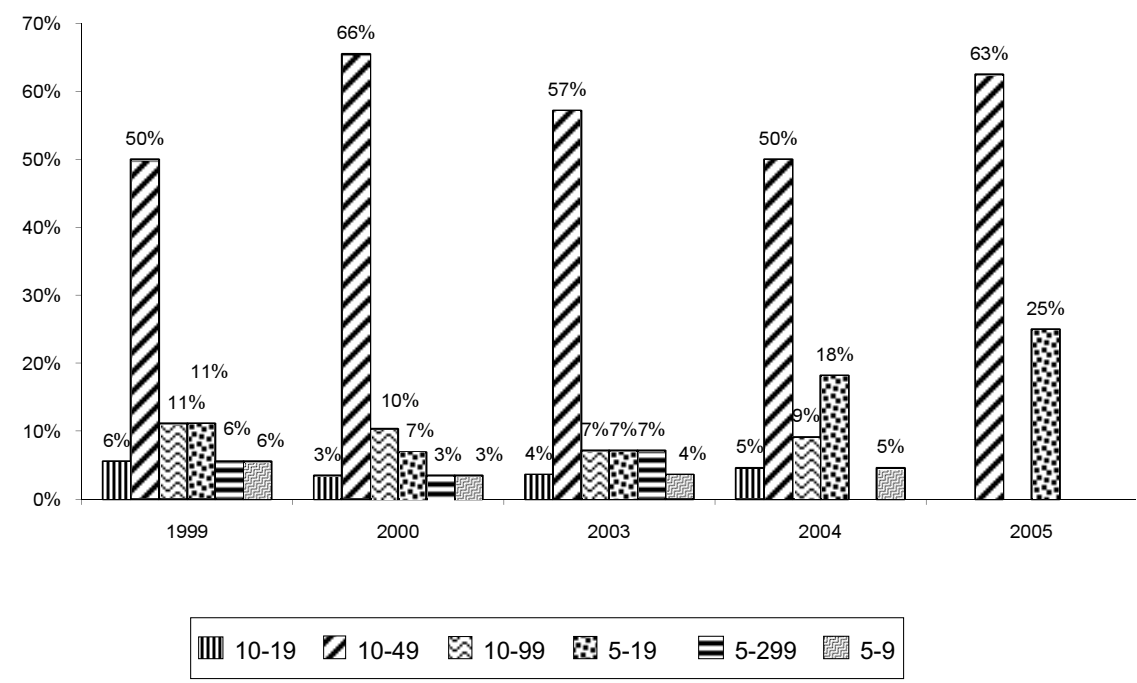

Fuente: "Micro, Small, and Medium Enterprises: A Collection of Published Data" 2007. Elaboración propia. 
más variados no demostrarían una política de desarrollo del sector tan homogénea.

En el gráfico 5 se muestra la importancia de las empresas de los sectores de la microempresa y la pequeña empresa en la generación del empleo. Es decir, muestra la contribución en la generación del total del empleo en cada país, como promedio en el periodo comprendido entre los años 1999 y 2005.

\section{Gráfico 5}

Empleo generado por el sector micro, pequeño y mediana empresa:

Promedio entre 1999-2005

(porcentajes)

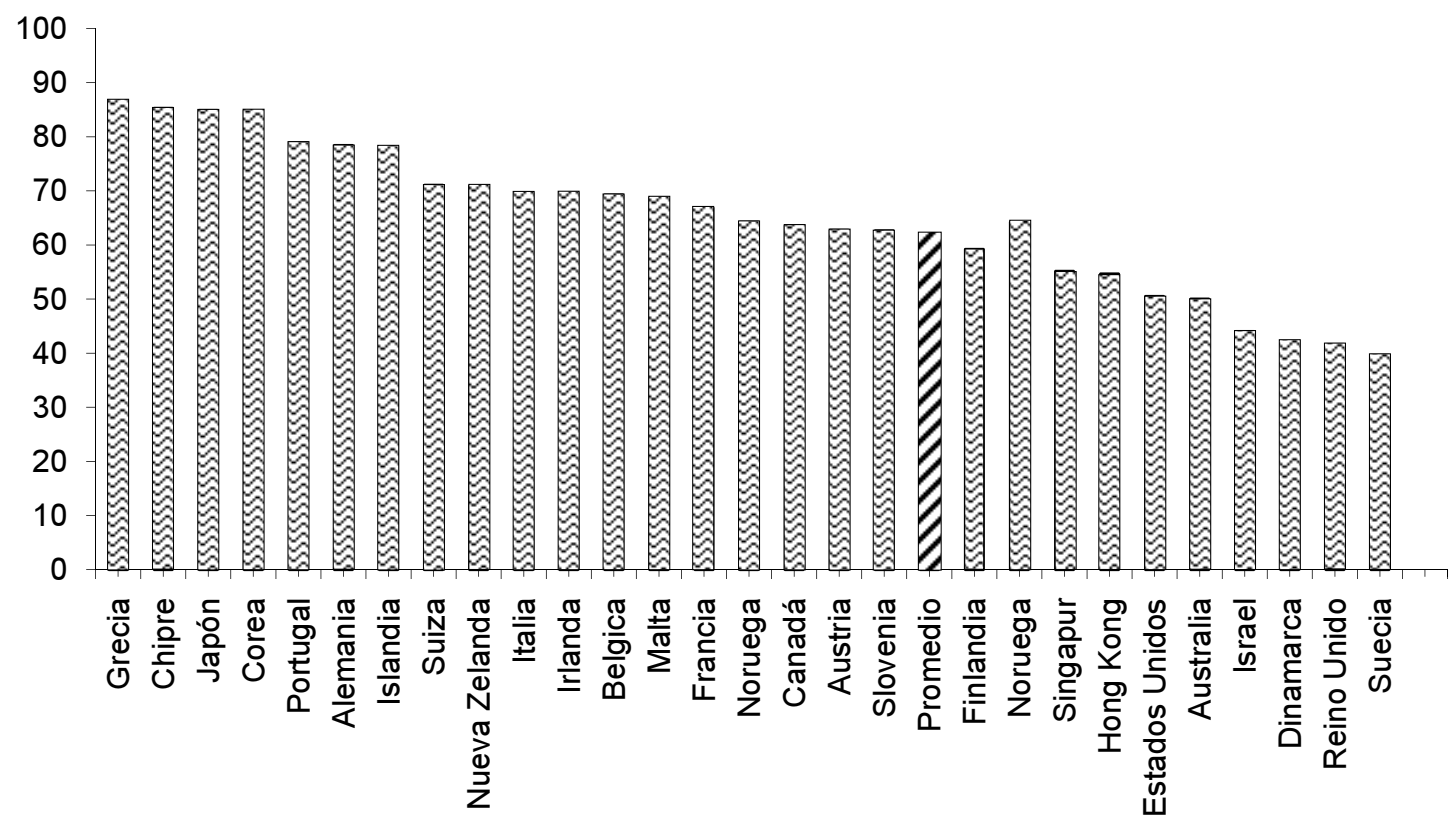

Fuente: "Micro, small and medium enterprises: A collection of published data" 2007. Elaboración propia. 
Una conclusión que podemos sacar de este gráfico es la gran importancia que tienen las pequeñas empresas y las microempresas al momento de generar empleo, y no solo en las economías de menores ingresos, como se esperaría. Podría considerarse erróneamente que las economías más grandes no tendrían un factor empleo tan importante; sin embargo, como se aprecia, el nivel promedio es cercano al $60 \%$ del empleo total, incluso en algunos países con niveles cercanos al $90 \%$ del total del empleo.

\subsection{Clasificación en países de ingresos bajos}

Como se aprecia en el gráfico 6, en este grupo de países la modalidad más utilizada es la que define a una microempresa como aquella en la que laboran hasta 9 personas, criterio bastante similar al que se presenta en el caso de los países de ingresos altos.

En cuanto a la generación de empleo, los resultados que se obtie-

\section{Gráfico 6}

Países de ingresos bajos: Definición de microempresa

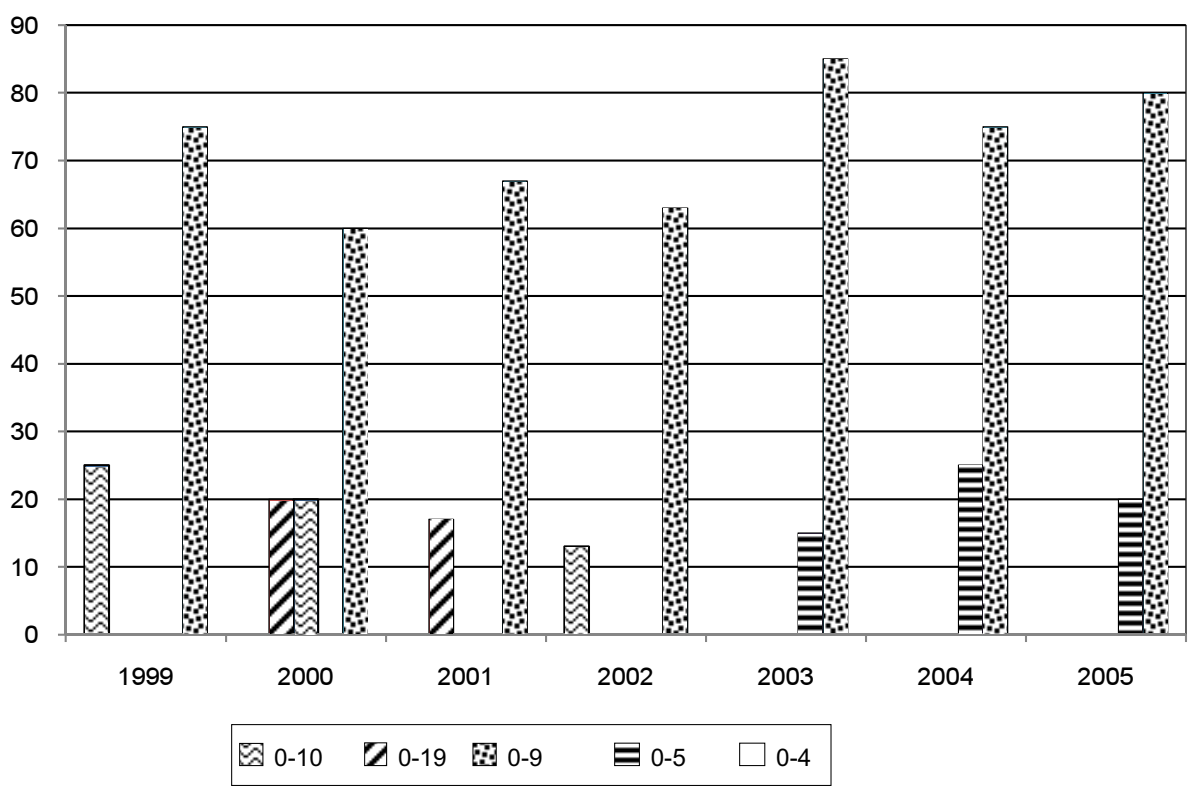

Fuente: "Micro, small and medium enterprises: A collection of published data" 2007.

Elaboración propia. 
nen del análisis de la base de datos utilizada no logran generar información suficiente para sacar una conclusión. En este caso, por ejemplo, los países clasificados como de ingresos bajos muestran unos reducidos niveles de generación de empleo. Esto puede deberse principalmente a la existencia de altos niveles de informalidad, que no permiten que la información del sector informal sea considerada dentro de la base de datos sustento de esta sección. Sin embargo, al comparar estos resultados con los obtenidos en los países de altos ingresos, puede apreciarse que al menos para la muestra tomada este subsector empresarial es el mayor generador de empleo.

\subsection{El ingreso per cápita y la generación de empleo por parte de las micro, pequeñas y medianas empresas}

En el gráfico 6 se pueden llegar a apreciar dos relaciones importantes en lo referente a la relación entre ingreso per cápita y generación de empleo por parte del sector micro, pequeña y mediana empresa. En primer lugar, podemos apreciar que existe una relación directa entre ingreso per cápita y generación de empleo en los países, pero esta se da solo hasta un cierto nivel de ingreso, que aproximadamente equivale a US\$10.000. Es decir, solo en los casos de países con niveles de ingreso per cápita altos se podría mostrar una relación inversa entre generación de empleo de las micro, pequeñas y medianas empresas. Este resultado se respalda en el hecho de que en los países de altos ingresos las empresas grandes llegan a determinar una porción importante en la generación de empleo, no solo de manera directa sino también indirecta. Otra conclusión interesante de este gráfico resulta del hecho de que la promoción de la creación de empresas definidas como pequeñas y microempresas redundarán en una mejora en la generación de empleo y en el ingreso per cápita. La anterior es una idea bastante arriesgada, ya que se estaría basando solo en el análisis gráfico de una data histórica, pero bien puede servir de punto de partida para un análisis más profundo en el futuro. 


\section{Gráfico 7}

Empleo generado por el sector micro, pequeña y mediana empresa: Países de bajos ingresos. Promedio entre 1999-2004

(porcentajes)

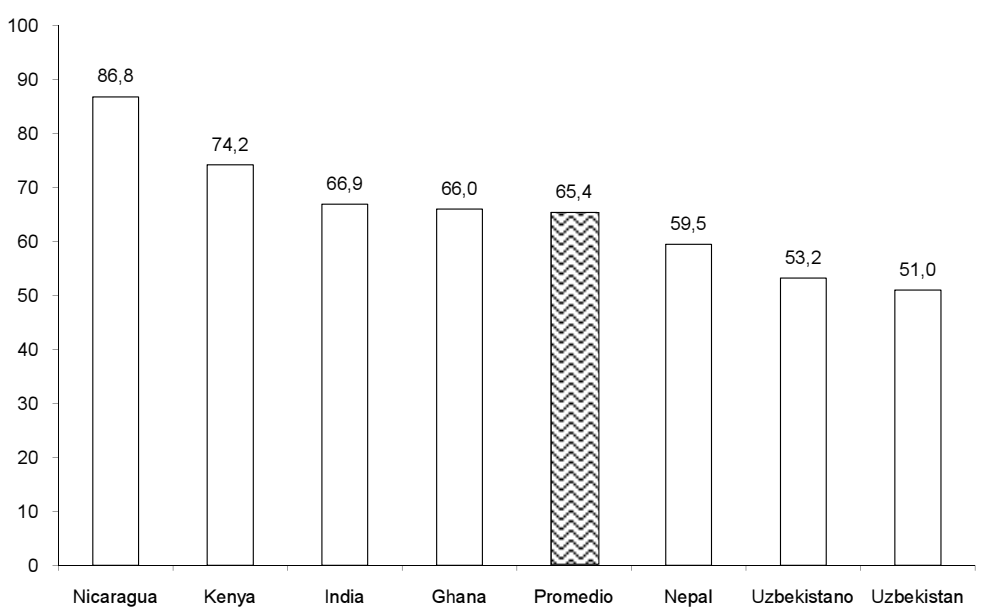

Fuente: "Micro, small and medium enterprises: A collection of published data" 2007. Elaboración propia.

\section{Gráfico 8}

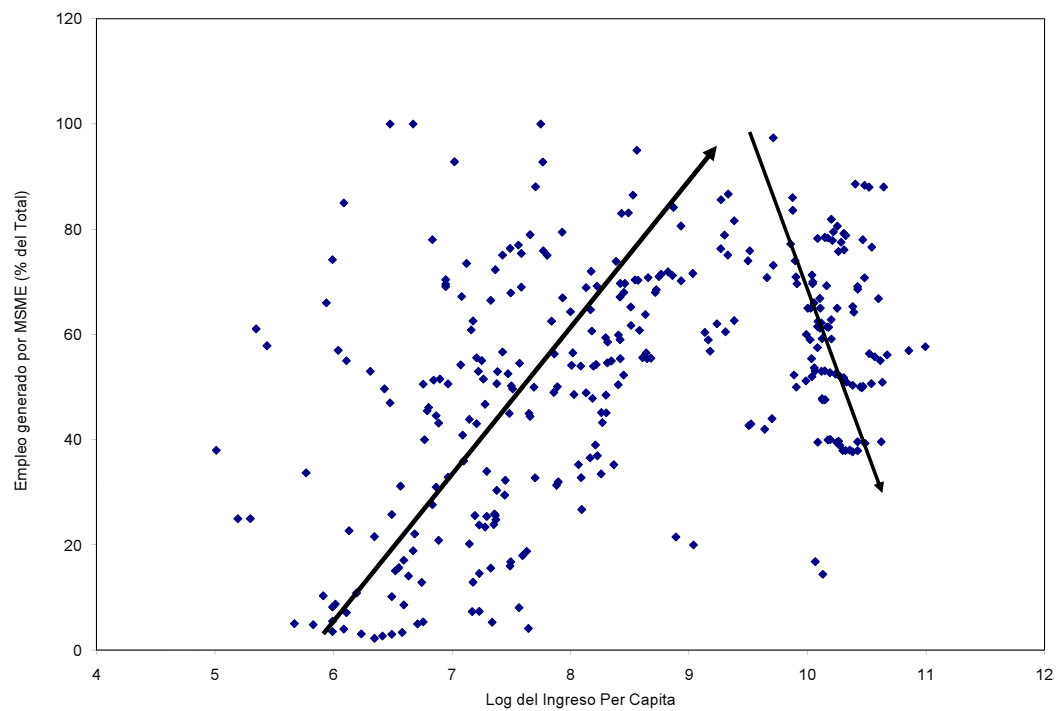

Fuente: "Micro, small and medium enterprises: A collection of published data" 2007. Elaboración propia. 


\section{CONCLUSIONES}

El resultado de esta investigación permitió llegar a las siguientes conclusiones:

- Las microfinanzas son un fenómeno que involucra no solo a los países pobres o de bajos ingresos.

- Existen grandes similitudes en las definiciones de microempresa a través de países con distintos niveles de ingreso; sin embargo, sí existe una mayor diversidad al momento de definir qué es una pequeña empresa. En general, la microempresa en su mayor parte son aquellas empresas que tienen entre 0 y 9 trabajadores. En el caso de las pequeñas empresas, al existir una mayor diversidad no se puede llegar a un consenso claro sobre su definición.

- Las microempresas y pequeñas empresas son agentes muy importantes al momento de la generación de empleo, principalmente en los países con niveles menores de ingresos, ya que la importancia de estas en la generación de empleo disminuye sobre todo en los países con ingresos per cápita elevados.

- La posición del Perú en el ámbito de desarrollo de las microfinanzas, medido a través de las variables de desempeño de las cajas municipales de ahorro y crédito, muestra que estamos frente a un sector que si bien crece de manera sostenida en el tiempo aún no alcanza su madurez, debido a que aún existe un mercado de demanda que exige cada vez más recursos, principalmente en un escenario de acelerado crecimiento como el vivido hasta el 2007.

- Este acelerado crecimiento de la economía se ha reflejado en una mayor capacidad de endeudamiento por parte de los clientes de las cajas municipales, en el caso del Perú. Internacionalmente, la capacidad de endeudamiento ha crecido de manera más lenta.

- Los resultados deben ser considerados en un escenario previo a la crisis financiera que se inició a fines del 2008. En ese escenario se logró apreciar una reducción en los niveles de morosidad, sin embargo esta situación podría variar, ya que el crecimiento del sector de micro y pequeñas empresas siempre reacciona de manera más volátil que la actividad económica. En ese sentido, el fortalecimiento patrimonial debería ser la mejor opción de las instituciones microfinancieras para poder afrontar los efectos de un incremento en la morosidad de su cartera de colocaciones. 


\section{BIBLIOGRAFÍA}

Armendáriz de Aghion, B. y J. Morduch (2000). "Microfinance beyond group lending". Economics of Transition. Vol. 8. Londres.

Banco Interamericano de Desarrollo (BID)-Corporación Andina de Fomento (CAF) (2007). El entorno de negocios para las microfinanzas en América Latina. Washington.

Banco Mundial (2007). "Micro, small and medium enterprises: A collection of published data". International Finance Corporation. $<$ http://rru.worldbank.org/Documents/Other/MSMEdatabase/msm e_companion_0706>.

Bancosol (2005). From microcredit to microfinance. La Paz: Bancosol.

Beck, J. y Ross Levine (2002). "Industry growth and capital allocation: Does having a market- or bank-based system matter?". Journal of Financial Economics.

Carvajal, Edgar y Paula Auerbach (2006). La microempresa en Ecuador: Perspectivas, desafíos y lineamientos de apoyo. Quito: Banco Interamericano de Desarrollo.

De Gregorio, José (2007). Macroeconomía. Teoría y políticas. México D. F.: Pearson.

Dirección General de Política de la Pyme (2007). Estadística Pyme. Informe estadístico 2006. Madrid.

García D. Jaime (2005). "Pymes, clusters y cadenas productivas". Ponencia presentada en ComexPerú.

Goldsmith, Raymond (1969). Financial structure and development. Yale: Yale University Press.

Gulli, Hege (1999). Microfinanzas y pobreza. ¿Son válidas las ideas preconcebidas? Washington: BID.

Gunterberg, Brigitte y Gunter Kayser (2004). SMEs (Small and medium-sized enterprises) in Germany. Facts and figures 2004. Bonn: Institut fur Mittelstandsforschung Bonn.

Instituto de Estadística (INE) - Directorio Central de Empresas (Dirce) de España (2008). Retrato de la pyme 2008. Madrid: Dirección General de Política de la pyme. 
Instituto de Estadística (INE)-Directorio Central de Empresas (Dirce) de España (2006). Estadistica española. Madrid.

Iniciativa Microfinanzas (2004). Reporte financiero de instituciones de microfinanzas a diciembre 2004. Lima: Copeme/USAID.

Iniciativa Microfinanzas (2005). Reporte financiero de instituciones de microfinanzas a diciembre 2005. Lima: Copeme/USAID.

Jaramillo B., M. y N. Valdivia (2005). Las políticas para el financiamiento de las pymes en el Perú. Lima: Grade/Cepal.

Khandler, Sahidur R. (abril del 2003). "Microfinanzas y pobreza: Evidencias en Bangladesh". Grameen Dialogue 54.

King, J. y Ross Levine (1993). "Finance and growth: Schumpeter might be right”. Quarterly Journal of Economics. Cambridge: The Quarterly Journal of Economics.

Levine, Ross y Zervos (1998). "Stocks markets, banks and economic growth". American Economic Review. Vol. 88, issue 3. Pittsburgh: American Economic Review.

Levine, Ross; Loayza, Norman y J. Beck (1999). "Financial intermediation and growth: Causality and causes". Journal of Monetary Economics. Nueva York: Elsevier.

Luna, E. y Ma. Victoria Sáenz (2004). Macroimpacto con microdinero: 25 años de apoyo a la microempresa. Washington: Banco Interamericano de Desarrollo.

Marulanda, Beatriz y María Otero (2005). The profile of microfinance in Latin America in 10 years: Vision \& characteristics. Boston: Accion International.

MicroBanking Bulletin. Benchmarks. Varios años. <http://www.themix. org/mbb/issues $>$.

Ministerio de Trabajo y Promoción del Empleo-Consejo Nacional para el Desarrollo de la Micro y Pequeña Empresa (2007). Actualización de estadisticas de la micro y pequeña empresa. Lima.

Ministerio de Trabajo y Promoción del Empleo-Prompyme (2005). La situación de la micro y pequeña empresa en el Perú. Lima. 
Morduch, J. (1999). "The microfinance promise". Journal of Economic Literature. Vol. 37, issue 4. Washington.

Navajas, S.; Navarrete, E.; Simbaqueba, L.; Cuevas, M. y G. Salamanca (2006). Indicadores de microfinanzas en América Latina: Rentabilidad, riesgo y regulación. Washington: BID.

Navajas, Sergio (2005). El grupo BID y la microempresa (1990-2004). Resultados de 15 años de trabajo. Washington.

OCDE (2004). SME statistics: Towards a more systematic statistical measurement of SME behaviour. Turquía.

Portocarrero Maisch, Felipe y Guillermo Byrne Labarthe (2003). Estructura de mercado y competencia en el microcrédito. Lima: Consorcio de Investigación Económica y Social (CIES).

Portocarrero, F.; Trivelli, M. y J. Alvarado Guerrero (2002). Microcrédito en el Perú: Quiénes piden, quiénes dan. Lima: CIES.

ProInversión y ESAN (2006). Mypequeña empresa crece. Guía para el desarrollo de la micro y pequeña empresa. Lima, 2006.

Romani Chocce, Gianni (2202). Impacto del microcrédito en los microempresarios chilenos: Un estudio de caso en la II Región de Chile. Anales del Congreso CLAD. Lisboa.

The Department for Business, Enterprise and Regulatory Reform (2007). Small and Medium-sized Enterprise (SME) Statistics for the UK 2006. Londres.

Villarán, F., Palacios, J.; Bullón, Víctor y T. Sanz (2005). Diagnóstico y recomendaciones para mejorar los programas y servicios de apoyo a las micro, pequeñas y medianas empresas (mipymes) en el Perú. Lima: Agencia Internacional de Desarrollo de los Estados Unidos (USAID).

Weiss, John; Montgomery, Heather y Elvira Kurmanalieva (2003). "Micro finance and poverty reduction in Asia: What is the evidence?". Asian Development Bank Institute. Research Paper Series 53.

Zevallos V., Emilio (2003). "Micro, pequeñas y medianas empresas en América Latina”. Revista de la Cepal 79. Santiago de Chile: Cepal. 